\title{
Fundus changes in high myopic Kashmiri population
}

\author{
Nazia Anjum 1 , Numrah Muqsit² ${ }^{2}$ Junaid S Wani ${ }^{3}$ \\ ${ }^{1}$ Senior Resident, Department of Ophthalmology PGIMER Chandigarh India, ${ }^{2}$ Senior Resident, ${ }^{3}$ Professor, Department \\ of Ophthalmology GMC Srinagar India
}

Background: High myopia (defined as myopia of $-6 \mathrm{D}$ or more) is one of the main causes of visual impairment worldwide. High myopia is always accompanied by pathological structural changes such as axial elongation, posterior staphyloma, lacquer crack formation, thinning of the retina and choroid, and choroidal neovascularization. Aims and Objectives: The purpose of this study was to examine the fundus changes in eyes with high myopia. Materials and Methods: All study participants underwent dilated fundus examination and fundus photography. Myopia-related macular (posterior staphyloma, lacquer cracks, Fuchs spot, myopic chorioretinal atrophy, and myopic choroidal neovascularization)and optic disc (optic nerve head tilt, optic disc dimensions, and peripapillary atrophy) changes were evaluated. Results: Statistical analysis was performed to evaluate fundus changes in eyes with high myopia. Data analysis included 107 eyes of 57 patients. Mean \pm SE was $12.07 \pm 3.184 \mathrm{D}$ in eyes with high myopia. Mean $\pm \mathrm{AL}$ was $26.68 \pm 1.577 \mathrm{~mm}$ in eyes with high myopia. The mean age was $28.54 \pm 9.44$ years $(14-50$ years). Fundus changes were: Temporal crescent in $56(52.33 \%)$ eyes, tessellated fundus appearance in $52(48.59 \%)$ eyes, lacquer cracks in $40(37.38 \%)$ eyes, tilted disc in $30(28 \%)$ eyes, lattice degeneration in $20(18.69 \%)$ eyes, posterior staphyloma in $20(18.69 \%)$ eyes, focal chorioretinal atrophy in $3(2.8 \%)$ eyes. CNV in $2(1.86 \%)$ eyes and retinal hole in $1(1 \%)$ eye. Conclusions: Tessellated fundus and temporal crescent were the most common fundus findings among Kashmiri population with high myopia. In this population, lacquer cracks and tilted disc were also common, while CNV and retinal holes were rare.

Key words: High Myopia; fundus changes; Kashmiri population
Access this article online

Website:

http://nepjol.info/index.php/AJMS

DOI: 10.3126/ajms.v12i1.31071

E-ISSN: 2091-0576

P-ISSN: 2467-9100

Copyright (c) 2021 Asian Journal of Medical Sciences

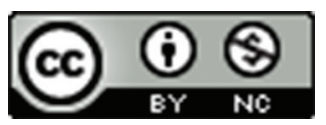

This work is licensed under a Creative Commons Attribution-NonCommercial 4.0 International License.

\section{INTRODUCTION}

High myopia (defined as myopia of $-6 \mathrm{D}$ or more) is one of the main causes of visual impairment worldwide. ${ }^{1}$ About $1 \%$ of the population suffers from this disease. ${ }^{2} \mathrm{High}$ myopia is always accompanied by pathological structural changes such as axial elongation, posterior staphyloma, lacquer crack formation, thinning of the retina and choroid, and choroidal neovascularization. ${ }^{3,45}$ One of the main causes of the ocular complications is excessive axial elongation of the eyeball.

The socioeconomic impact of blindness and visual impairment from myopia and high myopia is considerable, as it typically affects individuals during their productive years. ${ }^{6}$ In a clinic setting, a Japanese study of highly myopic individuals with greater than $-8.00 \mathrm{D}$ of spherical refractive myopia, several progressive patterns of pathologic myopia were seen - the initial finding of tessellated fundus, followed by staphyloma developing at approximately 40 years of age, and later with progression to diffuse atrophyand lacquer cracks. ${ }^{7}$ Although pathological myopia typically progresses with increasing age and high degree of myopia.

Our aim was to examine the pattern of myopia-related macular and optic disc changes in Kashmiri population with high myopia.

\section{MATERIAL AND METHODS}

It was a hospital based, observational, prospective study. The fundus changes of high myopic eyes were evaluated 
who attended the OPD of Postgraduate Department of Ophthalmology, Government Medical College, Srinagar. The study was conducted from September 2017 to September 2019. The study was approved by Ethical review committee.

In the high myopia group, the inclusion criteria were as follows: (1) high myopia was defined as SE > -6 diopters(D); (2) eyes with clear ocular media;

Patients were excluded from this study if they presented with any retinal abnormalities other than high myopia such as diabetic retinopathy, retinal vascular abnormalities, age related macular degeneration, laser or anti-VEGF therapy. Patients with the history of intraocular surgery, refractive surgery, glaucoma and amblyopia.

All the patients underwent refractive error examination without pupil dilatation using auto-refraction (VISUREF-100, make CARL ZEISS) and confirmed by manifest refraction. Spherical equivalent were calculated as the sum of spherical power and half of the cylinder. All eyes underwent a thorough ophthalmic evaluation, including intraocular pressure measurement (IOP/NCT - make AT 555), slit lamp biomicroscopy, dilated fundus examination (indirect ophthalmoscopy), fundus photography and axial length measurement (LENSTAR LS 900).

\section{Statistical analysis}

Data was entered in Microsoft Excel spreadsheet. Descriptive statistics including mean, standard deviation, minimum and maximum for continuous data and frequency and percentage for categorical variable.

\section{RESULTS}

A total of 107 eyes of highly myopic patients were included in the study. The mean age was $28.54 \pm 9.44$ years. The mean SE was $-12.07 \pm 3.18 \mathrm{D}$. The mean AL was 26.68 $\pm 1.57 \mathrm{~mm}$. The demographic and baseline characteristics are summarized in Table 1.

Fundus changes were: Temporal crescent in $56(52.33 \%)$ eyes, tessellated appearance in $52(48.59 \%)$ eyes, lacquer cracks in $40(37.38 \%)$ eyes, tilted disc in $30(28 \%)$ eyes, lattice degeneration in $20(18.69 \%$ ) eyes, posterior staphyloma in $20(18.69 \%)$ eyes, focal chorioretinal atrophy in $3(2.8 \%)$ eyes, CNV in $2(1.86 \%)$ eyes and retinal hole in $1(1 \%)$ eye (Table 2).

\section{DISCUSSION}

Among Kashmiri population with high myopia, temporal crescent $(52.33 \%)$, tessellated appearance $(48.59 \%)$, and

\begin{tabular}{lc} 
Table 1: Baseline demographic characteristics \\
\hline No. of eyes & 107 \\
No of eyes per males & 58 \\
No of eyes per females & 49 \\
Mean age & $28.54 \pm 9.44$ \\
Mean SE & $-12.07 \pm 3.18 \mathrm{D}$ \\
Mean AL & $26.68 \pm 1.57 \mathrm{~mm}$ \\
\hline
\end{tabular}

\begin{tabular}{lc} 
Table 2: Type of fundus changes \\
\hline Temporal crescent & $52.33 \%$ \\
Tessellated fundus & $48.59 \%$ \\
Lacquer cracks & $37.38 \%$ \\
Tilted disc & $28 \%$ \\
Lattice degeneration & $18.69 \%$ \\
Posterior staphyloma & $18.69 \%$ \\
Focal chorioretinal atrophy & $2.8 \%$ \\
CNV & $1.86 \%$ \\
Retinal hole & $1 \%$ \\
\hline
\end{tabular}

lacquer cracks $(37.38 \%)$, were common findings. Tilted disc $(28 \%)$, lattice degeneration $(18.69 \%)$ and posterior staphyloma $(18.69 \%)$ were also present. Focal chorioretinal atrophy $(2.8 \%), \mathrm{CNV}(1.86 \%)$ and retinal hole $(1 \%)$ were rare findings. These findings were similar to Singapore adult study. ${ }^{8}$ But in Singapore teenager study, staphyloma and chorioretinal atrophy were not present and only peripapillary atrophy and tilted discs were common in young subjects with high myopia. ${ }^{9}$

Elgouhary SM et al $(2017)^{10}$ reported the following findings in their study: myopic macular retinoschisis in 6 eyes $(6 \%)$, epiretinal membrane (ERM) in 6 eyes $(6 \%)$, myopic choroidal neovascularization (CNV) in 4 eyes (4\%), partial posterior vitreous detachment (PVD) in 4 eyes (4\%), foveoschisis in 2 eyes $(2 \%)$, myopic macular scars in 4 eyes ( $4 \%)$, macular hole in 4 eyes $(4 \%)$, retinal detachment in 2 eyes $(2 \%)$, combined findings in 31 eyes $(31 \%)$ and normal appearance in 43 eyes (43\%). Data was tabulated as number of findings and it's percentage of total cases as (mean, range and standard deviation) of best corrected visual acuity (BCVA), spherical equivalent of refraction and axial length (AL).

Thus, pathological myopia may be a disease that is dependent on the duration of disease and there is a lag phase from the onset of high myopia, often in the teenage years, to the presence of common macular lesions such as staphyloma and chorioretinal atrophy in middle-aged to elderly adults. In our study, only 2 eyes had CNV. Similarly, in Singapore study only 3 subjects had definite CNV. The rate of CNV was much higher in Japanese study, with more severe myopic refractive error seen in 91 eyes $(11.3 \%)$ at initial presentation. In our study, there was no retinal detachment in any subject. This may be due to our sample size was small. In our study, choroidal thickness was 
measured by OCT with EDI mode and there was increased thinning of choroid with increase in degree of myopia.

Fundus changes observed in high myopia include tigroid fundus (choroidal vessels being visible through the retina, often with lacquer cracks in Bruch's membrane), geographical atrophy of the retinal pigment epithelium (RPE) and choroid (diffuse or patchy), posterior staphyloma, and choroidal neovascularization (CNV) that is also referred to as Fuch's spot at its later stage. ${ }^{11}$

\section{LIMITATIONS OF THE STUDY}

The study was cross-sectional in nature and the temporal sequence of progression of lesions could not be documented. Secondly the sample size was small.

\section{CONCLUSION}

Tessellated fundus and temporal crescent were the most common fundus findings in Kashmiri population with high myopia while $\mathrm{CNV}$ and retinal holes were rare findings.

\section{ACKNOWLEDGEMENT}

The authors take this opportunity to thank Department of Ophthalmology, GMC Srinagar to carry out the present study.

\section{REFERENCES}

1. Klaver CC, Wolfs RC, Vingerling JR, Hofman A and de Jong PT. Age specific prevalence and causes of blindness and visual impairment in an older population: the Rotterdam Study. Arch Ophthalmol. 1998; 116: 653-658.

https://doi.org/10.1001/archopht.116.5.653
2. Vongphanit J, Mitchell $\mathrm{P}$ and Wang JJ. Prevalence and progression of myopic retinopathy in an older population. Ophthalmology. 2002; 109: 704-711. https://doi.org/10.1016/S0161-6420(01)01024-7

3. Lim MC, Hoh ST, Foster PJ, Lim TH, Chew SJ, Seah SK, et al. Use of optical coherence tomography to assess variations in macular retinal thickness in myopia. Invest Ophthalmol Vis Sci. 2005; 46: 974-978. https://doi.org/10.1167/iovs.04-0828

4. Curtin BJ and Karlin DB. Axial length measurements and fundus changes of the myopic eye. Am J Ophthalmol. 1971; 71: 42-53. https://doi.org/10.1016/0002-9394(71)91092-0

5. Takano $M$ and Kishi $S$. Fovealretinoschisis and retinal detachment in severely myopic eyes with posterior staphyloma. Am J Ophthalmol. 1999; 128: 472-476. https://doi.org/10.1016/S0002-9394(99)00186-5

6. Vongphanit J, Mitchell $\mathrm{P}$ and Wang JJ. Prevalence and progression of myopic retinopathy in an older population. Ophthalmology. 2002; 109(4): 704-711.

https://doi.org/10.1016/S0161-6420(01)01024-7

7. Hayashi K, Ohno-Matsui K, Shimada N, Moriyama M, Kojima A, Hayashi W, et al. Long-term pattern of progression of myopic maculopathy: a natural history study. Ophthalmology. 2010; 117(8): 1595-1611. https://doi.org/10.1016/j.ophtha.2009.11.003

8. Chang L, Pan CW, Ohno-Matsui K, Lin X, Cheung GCM, Gazzard G, et al. Myopia-related fundus changes in Singapore adults with high myopia. Am J Ophthalmol. 2013; 155: 991-999. https://doi.org/10.1016/j.ajo.2013.01.016

9. Samarawickrama C, Mitchell P, Tong L, Gazzard G, LimL, WongTY, et al. Myopia-related optic disc and retinal changes in adolescent children from Singapore. Ophthalmology. 2011;118:2050-2057. https://doi.org/10.1016/j.ophtha.2011.02.040

10. Elgouhary SM, El Sebaey Sarhan AR, Ibrahim AM and EI Din Abd El Hi LAG. Macular Findings in Highly Myopic Eyes Using OCT and its Correlation to Refractive State, Axial Length and Visual Acuity. International Journal of Ophthalmic Research. 2017; 3(2): 226-230. https://doi.org/10.17554/j.issn.2409-5680.2017.03.55

11. Chan CK and Lawrence FC. Macular hole after laser in situ leratomileusis and photorefractive keratectomy. Am J Ophthalmol. 2001; 131: 666-667. https://doi.org/10.1016/S0002-9394(00)00855-2

\footnotetext{
Author's Contribution:

NA-Concept and design of the study; interpreted the results, prepared first draft of manuscript, critical revision of the manuscript and coordination of the overall study; NM- Statistically analyzed and interpreted; reviewed the literature and revision of manuscript; JSW- Design of the study, preparation of manuscript and revision of manuscript.

Work attributed to:

Department of Ophthalmology, GMC Srinagar, Jammu and Kashmir, India.

Orcid ID:

Dr. Nazia Anjum- it https://orcid.org/0000-0002-5669-1870

Dr. Numrah Muqsit- (1) https://orcid.org/0000-0002-0895-7929

Dr. Junaid S Wani- (1) https://orcid.org/0000-0003-1724-5453

Source of funding: Department of Ophthalmology, GMC Srinagar, Conflict of interest: None.
} 